\title{
Delay in presentation of patients with rheumatoid arthritis for specialty care
}

\author{
Dandeniya $\mathrm{CL}^{1}$, Rubasinghe $\mathrm{J}^{2}$ \\ Journal of the Ceylon College of Physicians, 2021, 52, 101-104
}

\begin{abstract}
Introduction: Early diagnosis and intervention is known to improve the outcomes in patients with rheumatoid arthritis. Information on the time duration from the symptom onset of rheumatoid arthritis to the presentation to specialty care in Sri Lanka is not available. The aim of this study was to evaluate the time duration from symptom onset to the first visit of patients to a rheumatology specialty clinic in Sri Lanka and changing trends over a period.
\end{abstract}

Methods: This retrospective observational study was carried out in the rheumatology and rehabilitation clinic at the National Hospital of Sri Lanka. Data were collected through an interviewer-administered questionnaire from 60 consecutive consenting patients with rheumatoid arthritis attending the clinic over a period of one month. Further information was added or clarified by perusing clinic records. Data was analyzed using SPSS software version 17.0 and Microsoft Excel 2010.

Results: Sixty patients were recruited to the study. Their mean age was 54.23 years (range 19-76 years) and 58 were women. Among them, 38 patients were positive for either rheumatoid factor or anti-cyclic citrullinated peptide and were considered as seropositive. The average time duration from symptom onset to the first visit to the rheumatology clinic was 15.86 months $(S D=18.38)$. The analysis of trends over 13 years showed an improving trend with gradually shortening time duration. There was no statistically significant difference in the time lag of the first visit to the rheumatology clinic between seronegative and sero-positive groups ( $p=0.976 . \mathrm{Cl} 95 \%)$.

Conclusion: There was a significant delay in presentation of patients with rheumatoid arthritis to specialty care, with a trend of gradual improvement over recent years. For optimum treatment outcome, referral of suspected cases to specialty care within 6 weeks of presentation to the first medical contact is recommended. We found that there is a significant delay in referral system that needs remedying to meet the standard recommendations.

Key words: rheumatoid arthritis, delay in presentation, early referral

\section{Introduction}

Rheumatoid arthritis is a debilitating chronic disorder with high morbidity. ${ }^{1,2}$ With recent advances in rheumatology, more emphasis is placed on early diagnosis since early intervention leads to better outcomes. ${ }^{1,3,4}$ Therefore, the international classification criteria for rheumatoid arthritis has amended the required duration of symptoms for diagnosis from 6 months in $1987^{5}$, to 6 weeks in $2010 .{ }^{6}$ The European League Against Rheumatism (EULAR) emphasizes that the diagnosis of rheumatoid arthritis remains clinical and can even be made before six weeks at the discretion of the rheumatologist. ${ }^{4}$ There is a recent interest in research focusing on the diagnosis and management of 'early inflammatory arthritis'. The

\footnotetext{
${ }^{1}$ Consultant in Rheumatology and Rehabilitation, Teaching Hospital, Peradeniya, Lecturer, Department of Medicine, Faculty of Medicine, University of Peradeniya, Sri Lanka, ${ }^{2}$ Consultant in Rheumatology and Rehabilitation, Department of Rheumatology and Rehabilitation (Special Unit), National Hospital of Sri Lanka, Colombo, Sri Lanka.
}

Correspondence: CLD, e-mail: chathurika.dandeniya@med.pdn.ac.lk

https://orcid.org/0000-0001-8857-1366

Received 28 August 2021, accepted 05 November 2021

This is an open-access article distributed under the terms of the Creative Commons Attribution License, which permits unrestricted use, distribution, and reproduction in any medium, provided the original author and source are credited. 
disease modifying treatment should be started as early as possible, preferably within three months, in persistent arthritis even if they do not fulfill definite classification criteria for inflammatory arthritis. ${ }^{4}$ Given the difficulty in diagnosing early inflammatory arthritis and the challenges of treatment, it is recommended that early arthritis should be managed under the care of a rheumatologist. ${ }^{4}$

It is a personal observation that a delay in presentation of patients to specialty rheumatology clinics is common in Sri Lanka. However, this observation has not been investigated in a scientific study. The findings of such a study would help in patient education and for the future development of a national referral policy in rheumatology care in the country. The aim of this study was to investigate the time duration from the onset of symptoms to the first visit to a specialized rheumatology clinic and the changing trends over time.

\section{Methods}

This cross-sectional retrospective study was conducted at the department of rheumatology and rehabilitation of the National Hospital of Sri Lanka. Ethical approval was obtained from the ethical review committee of the National Hospital of Sri Lanka. All consecutive patients with rheumatoid arthritis followed up in the unit over a period of one month (September 2018), who consented to the study, were included. Data was collected by the first author (CD) through an interviewer-administered questionnaire and by perusal of clinic records. Demographic details, final diagnosis according to the serological status and the symptom duration at the confirmation of diagnosis were recorded. The symptom duration prior to first clinic visit, after being extracted from the records, was verified by the patients. All patients had a diagnosis fulfilling the classification criteria set by the American College of Rheumatology $(1987)^{5}$ or the American College of Rheumatology/EULAR joint statement $(2010)^{6}$ at the time of diagnosis. Those patients with a diagnosis not conforming to the above classification criteria were excluded. Further, those with incomplete records at the time of diagnosis and those with overlap syndromes were excluded from the study. Data were analyzed using SPSS software version 17.0 and Microsoft Excel 2010.

\section{Results}

\section{Demographic characteristics}

A total of sixty patients were recruited to the study. Of them, 58 were women with a female: male ratio of 29: 1 . The mean age was 54 years (range 19-76 years) at the time of diagnosis (Figure 1). Duration of rheumatoid arthritis at the time of data collection ranged from 2 months to 31 years. Of the patients, 22 were diagnosed with sero-negative rheumatoid arthritis and 38 were sero-positive. Sero-positivity was defined as being positive for rheumatoid factor, anti-citrullinated cyclic peptide or both.

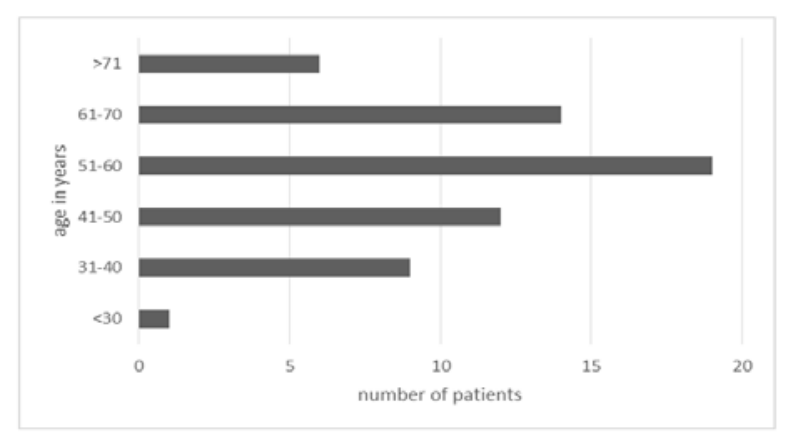

Figure 1. Age distribution of the patients at the recruitment to the study.

Time lag was defined as the time from the onset of symptoms to the first visit to a specialized rheumatology clinic. The mean of time lag was 15.86 (SD 18.38) months. The mean time lag among the seronegative group was 15.77 (SD 19.42) months and in the sero-positive group was 15.92 (SD 18.02) months. Applying independent 2 sample t test, where equal variance was assumed after performing Levene's test for equality of variances, no significant difference was noted between the average time lag of the two groups $(P=0.976$. $\mathrm{Cl}$ 95\%).

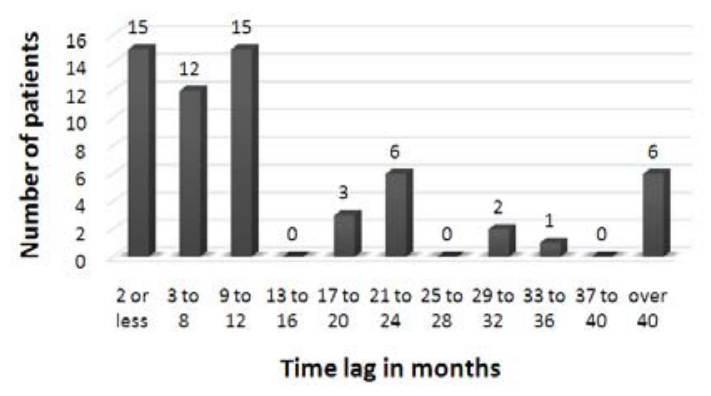

Figure 2. The time lag (months) and the number of patients.

The time lags were further analyzed and depicted in figure 2. This shows that $25 \%(n=15)$ of the patients presented to specialty care within two months of symptom onset while $30 \%(n=18)$ presented more than 12 months after symptom onset. The remaining 45\% $(n=27)$ presented within 2 to 12 months of symptom onset. 
The trend of the time lag to the first visit to specialty care in the study population is shown against the year of first visit in Figure 3 . This shows that the average delay in referral is decreasing and has reached 7.37 months (SD 5.5) by 2015 to 2018.

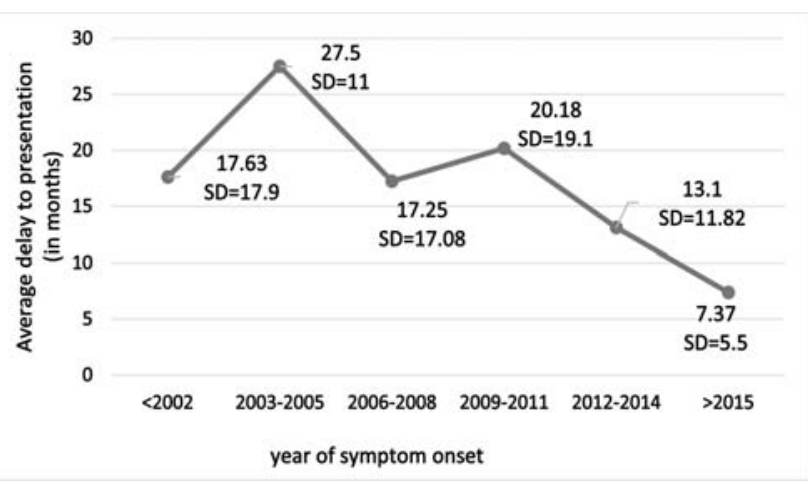

Figure 3. Trend in the time lag of patients with rheumatoid arthritis presenting to specialist care over the years.

\section{Discussion}

This single centre study in Sri Lanka showed that there was a significant delay in presentation of patients with rheumatoid arthritis to specialty care. Further analysis of data revealed that the delay showed an improving trend over time. There was no significant difference in the time to the first visit to specialty care between sero-positive and sero-negative groups. Given the international standard of care aiming for treatment initiation within 6 weeks of symptom onset, there is a timely need for further improvement.

With an average delay of 15.86 months (SD 18.38) from symptom onset to the first visit to specialty care, there was a significant delay in initiating treatment for rheumatoid arthritis in this study population. However, statistically, the standard deviation exceeding the mean may reflect a wide range of data or a skewed distribution. Removing one extreme outlier from the final analysis did not make a significant difference to the outcome.

To correct for the wide variation of data, further analysis was done clustering the lag time to the first visit in months. This showed a wide variation in the time delay ranging from less than 2 months from symptom onset to more than 12 months. Although the average delay to presentation was long, this showed that $25 \%$ of patients presented to specialty care within 2 months of symptom onset and another $45 \%$ presented within 2 to 12 months. But a delay of more than 12 months was observed in another 30\% of the cohort. By identifying the reasons contributing to this early versus delayed presentation, a targeted approach may be designed to overcome this.

Considering the possibility of a skewed distribution, we considered a second cluster analysis, depending on the year of first visit to specialty care, and removal of extreme outliers within subgroups. This revealed the improving trend of referral over time, with gradual reduction of time from symptom onset to the first visit to specialty care. With the wider availability of specialty rheumatology services, referral would have become more acceptable.

Another important observation was that there was no significant difference in the average time lag between sero-positive and sero-negative patients $(p=0.976$. Cl 95\%). In a large-scale cohort study done in the United Kingdom, sero-positive patients presented later to primary care than sero-negative patients. However, referral from primary care to specialist care was delayed in the sero-negative group, and once referred, initiation of treatment was also delayed. ${ }^{7}$ The authors postulated that the delay of seropositive patients in presentation to primary care was likely to be the result of more insidious onset of symptoms. ${ }^{7}$ Further, the sero-positive group was younger and may have had distinct health seeking behavior patterns. ${ }^{7}$ False reassurance given to the primary care doctors by negative antibodies as well as the established referral guidelines including antibody positivity may have contributed to the delay in referral of sero-negative patients to secondary care. ${ }^{7}$ Once referred to secondary care, the delay in initiation of treatment, was attributed to diagnostic difficulty in sero-negative arthritis $^{7}$. In our study, we assessed the time lag at specialty clinic level, a level equivalent to secondary care in the above study, and no difference between the two groups was noted.

Research has unequivocally shown that earlier diagnosis and institution of disease modifying drugs improve the outcome in rheumatoid arthritis ${ }^{1,4,8}$. Evidence to-date suggests that rheumatoid arthritis is better managed by rheumatologists due to several reasons: earlier diagnosis and experience in using and adjusting disease modifying anti-rheumatoid drugs (DMARDs). ${ }^{4,9}$

Although there is an improving trend over the years, the target should be for the patients to be reviewed in specialty clinics at least within 6 weeks of the presentation to the first medical contact. Although Sri Lanka has a well-established secondary care system there is a lack of clearly defined referral pathways from 
the primary care level. In the recent years, there has been an increase in the availability of rheumatology services in secondary care level which can contribute to improved care. To reach the international standards of care in managing early rheumatoid arthritis, early diagnosis, referral and initiation of treatment have to be prioritized.

\section{Limitations}

There are several limitations in our study. This study was a cross-sectional study carried out in patients already diagnosed with rheumatoid arthritis and followed up in the clinic over several years. Therefore, we had to rely on the accuracy of the clinic records and the patient memory to verify the time duration from symptom onset to presentation. This could be overcome by a prospective study of newly referred patients to the rheumatology clinic.

Another limitation is the generalizability of the data given the study setting. As the National Hospital of Sri Lanka is a tertiary referral center, this data may not represent the situation of the country in general. However, given the less accessibility of services towards the peripheries, the average lag to presentation of patients with rheumatoid arthritis may actually be longer. Therefore, the learning point from the study would remain valid.

\section{Conclusion}

There is a significant delay in presentation of patients with rheumatoid arthritis to specialty care, with a trend of gradual improvement over recent years. For optimum treatment outcome, the aim should be for referral of suspected cases to specialty care within 6 weeks of presentation to the first medical contact. An organized national policy on referral and long-term care is a timely need.

\section{Acknowledgements}

The authors wish to acknowledge Professor S.A.M. Kularatne (Department of Medicine, University of Peradeniya) for his help in the preparation of the manuscript, the patients for participation, and all tiers of staff in the rheumatology and rehabilitation clinic (special unit) of the National Hospital of Sri Lanka for their support.

\section{Competing interests}

Authors declare no conflicts of interest.

\section{Contribution of the authors}

CLD formulated the concept of the study, designed the study, recruited patients, collected and analyzed data and wrote the final manuscript. JR oversaw the design of the study, recruited patients and revised and edited the manuscript.

\section{References}

1. van der Linden MPM, le Cessie L, Raza K, et al. Long-Term Impact of Delay in Assessment of Patients with Early Arthritis. Arthritis \& Rheumatism 2010; 62(12): 3537-46.

2. Matteson EL, Mankad R. Assessment of Cardiovascular Risk in Patients with Rheumatoid Arthritis. J Rheumatol. 2016; 43(11): 1947-9.

3. Combe B, Landewe R, Lukas C, et al. EULAR recommendations for the management of early arthritis: report of a task force of the European Standing Committee for International Clinical Studies Including Therapeutics (ESCISIT). Ann Rheum Dis. 2007; 66: 34-45.

4. Combe B, Landewe R, Daien $\mathrm{Cl}$, et al. 2016 update of the EULAR recommendations for the management of early arthritis. Ann Rheum Dis. 2017; 76: 948-59. doi:10.1136/annrheumdis-2016-210602

5. Arnett FC, Edworthy SM, Bloch DA, et al. The American Rheumatism Association 1987 revised criteria for the classification of rheumatoid arthritis. Arthritis Rheum. 1988; 31: 315-324.

6. Aletaha D, Neogi T, Silman AJ, et al. 2010 Rheumatoid arthritis classification criteria: An American College of Rheumatology/ European League Against Rheumatism collaborative initiative. Arthritis \& Rheumatism. 2010; 62: 2569-81. doi:10.1002/art.27584

7. Pratt AG, Lendrem D, Hargreaves B, Aslam O, Galloway JB, Isaacs JD. Components of treatment delay in rheumatoid arthritis differ according to autoantibody status: validation of a single-centre observation using national audit data. Rheumatology 2016; 55: 1843-48.

8. Sørensen J, Hetland ML. Diagnostic delay in patients with rheumatoid arthritis, psoriatic arthritis and ankylosing spondylitis: results from the Danish nationwide DANBIO registry. Ann Rheum Dis. 2015; 74: e12.

9. Smolen JS, Landewé R, Breedveld FC, et al. EULAR recommendations for the management of rheumatoid arthritis with synthetic and biological disease-modifying antirheumatic drugs: 2013 update. Ann Rheum Dis. 2014; 73: 492-509. 